\title{
Live Demonstration: Quasi-Digital Portable Pen to Monitor Anaesthetics Delivery
}

\author{
Simone Aiassa*†, Francesco Grassi*†, Rossana Terracciano*, Sandro Carrara ${ }^{\dagger \ddagger}$, and Danilo Demarchi* ${ }^{* \ddagger}$ \\ ${ }^{*}$ Department of Electronics and Telecommunications, Politecnico di Torino, Turin, Italy \\ †Integrated Systems Laboratory, École Polytechnique Fédérale de Lausanne, Lausanne, Switzerland \\ ${ }^{\ddagger}$ Integrated Circuits Laboratory, École Polytechnique Fédérale de Lausanne, Neuchâtel, Switzerland \\ Email: simone.aiassa@polito.it
}

\begin{abstract}
The live demonstration presents the innovative concept of a portable pen-device able to sense anaesthetic compounds over time for improved anaesthesiology practice. A custom Printed Circuit Board (PCB) manage in the event-based quasidigital domain the sampling procedure, while a commercial Screen Printed Electrode (SPE) acts as an electrochemical sensor for measuring paracetamol (APAP) as benchmark drug. The system is controlled by an FPGA board implementing a custom logic and the results are displayed through a Matlab ${ }^{\circledR}$ graphical user interface. The visitors can experience and confirm on real-time the reliability of the here presented portable pen for anaesthetic monitoring.
\end{abstract}

\section{INTRODUCTION}

The anaesthesia is a critical medical procedure that requires the administration of a cocktail of drugs: hypnotic, analgesic, and muscle relaxant. The usage of prediction models to estimate the right dosage presents today high errors due to patients diversity. Differently, measuring the actual drug concentration in the patients body is possible to meet the personal requirements with a dynamic adjustment. To overcome the lack of commercially-available systems to monitor on realtime the anaesthetics, we here propose a portable-pen able to measure at the point-of-care the delivery-rate of therapeutic compounds. The design of the system is based on a bioinspired event-based approach to guarantee low complexity, low power consumption, and reduced noise, while it implements Voltammetry Based Sensing (VBS) techniques [1]. The presented approach obtains comparable performances in terms of sensitivity and resolution with the ones obtained by expensive commercial instrumentation [2].

\section{Demonstration Setup}

In this demonstration (Fig 1), pre-prepared samples containing acetaminophen (APAP) as benchmark drug are analysed in real-time through the proposed portable-pen. Namely, the commercial Screen-Printed Electrode (SPE) acting as biosensor is immersed in $10 \mathrm{ml}$ becher containing Phosphate Buffer Saline (PBS) and a known concentration of APAP. The portable-pen perform a Cyclic Voltammetry (CV) electrochemical procedure. A custom digital interface deployed on an FPGA board transmits the data to a laptop via UART

This work is supported by Politecnico di Torino and Compagnia di San Paolo under the initiative "Joint research projects with top universities".

Demonstration video link: https://youtu.be/7JL_vAfjIrY

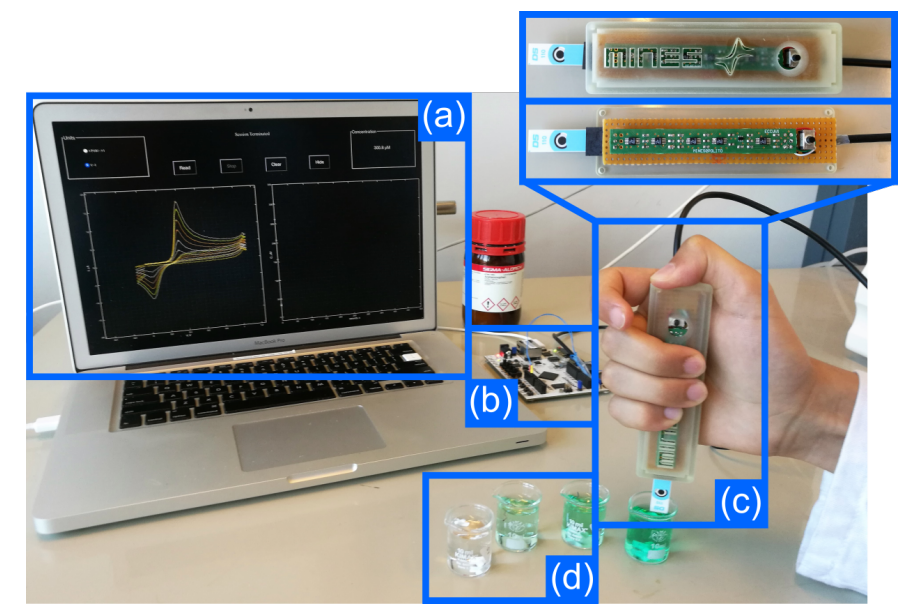

Fig. 1: Demonstration setup, GUI display interface (a), FPGA control logic (b), proposed portable pen (c), APAP samples (d).

communication. The laptop will display the results in realtime of the APAP detection through a Matlab ${ }^{\circledR}$ Graphical User Interface (GUI), highlighting the oxidation current peak and providing to the visitor step by step the concentration monitored by the proposed system.

\section{VISITOR EXPERIENCE}

The visitors will experience both the portability and effectiveness of our anaesthetics monitoring pen, meanwhile, they will appreciate the capability of the event-based quasi-digital design approach. In the demonstration, the visitor will also face the methods performed to detect drugs through direct VBS techniques with live insights on both the procedure and the data processing.

\section{REFERENCES}

[1] S. Aiassa, S. Carrara, and D. Demarchi, "Optimized sampling rate for voltammetry-based electrochemical sensing in wearable and IoT applications," IEEE Sensors Letters, vol. 3, no. 6, pp. 1-4, 2019. [Online]. Available: https://ieeexplore.ieee.org/document/8721138

[2] S. Aiassa, F. Stradolini, A. Tuoheti, S. Carrara, and D. Demarchi, "QuasiDigital Biosensor-Interface for a Portable Pen to Monitor Anaesthetics Delivery", 2019 15th Conference on Ph.D. Research in Microelectronics and Electronics (PRIME), Lausanne, Switzerland, 2019, pp. 265-268. [Online]. Available: https://ieeexplore.ieee.org/document/8787764 